\title{
Ageing studies of TPB in noble gas detectors for dark matter and neutrinoless $\beta \beta$ decay searches
}

\author{
N. Yahlali a,*, J.M. Garcia ${ }^{\text {a }}$, J. Díaz ${ }^{\text {a }}$, A. Soriano ${ }^{\text {b }}$, L.M.P. Fernandes ${ }^{c}$ \\ a Instituto de Física Corpuscular (IFIC), CSIC E Universitat de València, Catedrático José Beltrán 2, 46980 Paterna, Valencia, Spain \\ b Instituto de Ciencia Molecular (ICMOL), Universitat de València, Catedrático José Beltrán 2, 46980 Paterna, Valencia, Spain \\ c LIBPhys, Department of Physics, University of Coimbra, 3004-516 Coimbra, Portugal
}

\section{A R T I C L E I N F O}

\section{Article history:}

Received 14 October 2015

Received in revised form 3 March 2016

Accepted 8 April 2016

Available online 14 April 2016

\section{Keywords:}

Neutrinoless double-beta decay

High pressure xenon gas

Time projection chamber (TPC)

Electroluminescence

Wavelength shifter

\begin{abstract}
A B S T R A C T
Noble gases (Xe, $\mathrm{Ar}, \mathrm{Kr}$ ) are very attractive as detector media in Dark Matter search and neutrinoless double-beta decay experiments. However, the detection of their scintillation light (in the VUV spectral region) requires shifting the VUV light to visible light, where standard photosensors are more efficient. Tetraphenyl butadiene (TPB) is widely used as wavelength shifter, absorbing the VUV light and re-emitting in the blue region $(\sim 430 \mathrm{~nm})$. TPB is an organic molecule that may degrade due to exposure to environmental agents and also to ultraviolet light. In this work, we present TPB ageing studies due to exposure to VUV light, aiming at quantifying the reduction of the absolute fluorescence yield of TPB coatings of several thicknesses $(130 \mathrm{~nm}, 260 \mathrm{~nm}, 390 \mathrm{~nm}$, $1600 \mathrm{~nm}$ ), exposed to various doses of VUV light at $170 \mathrm{~nm}$ (similar to the Xe scintillation). In our setup, the VUV light is produced from a vacuum monochromator coupled to a deuterium lamp. The VUV exposure in our setup is compared to the exposure obtained in the electroluminescent gaseous Xe TPC of the NEXT-100 experiment for neutrinoless double-beta decay search.
\end{abstract}

@ 2016 Elsevier B.V. All rights reserved.

\section{Introduction}

Heavy noble gases (argon, krypton, xenon) have outstanding spectroscopic properties that make them very attractive as detector media in Dark Matter search and neutrinoless double-beta $(\beta \beta)$ decay experiments [1]. However, their scintillation light, which is in the vacuum ultraviolet (VUV) spectral region, is difficult to detect by most standard photodetectors, especially silicon photomultipliers (SiPM), which are mostly efficient in the visible spectral region. The optical readout of noble gas detectors thus requires shifting the VUV scintillation to visible light using a wavelength-shifter. The organic compound fluor tetraphenyl butadiene (TPB) of $\geq 99 \%$ purity grade [2] absorbs light in the VUV-UV spectral region and reemits in the blue region, around $430 \mathrm{~nm}$, as seen in Fig. 1. TPB is used as a wavelength shifter in noble gas detectors (NEXT [3], ArDM [4]) due to the short scintillation wavelengths of the noble gases ( $128 \mathrm{~nm}$ for $\mathrm{Ar}, 172 \mathrm{~nm}$ for Xe). It is used as a coating for the active surface of the photosensors and the internal walls of the detectors.

TPB is an organic molecule known to degrade when exposed to environmental agents (mostly humidity and oxygen) and to intense light levels, by means of free-radical mediated photo-oxidation reactions [5]. This degradation results in a reduction of the fluorescence yield of the molecules and the subsequent reduction of the

\footnotetext{
* Corresponding author.

E-mail address: Nadia.Yahlali@ific.uv.es (N. Yahlali).
}

signal amplitudes in noble gas detectors. However, to our knowledge, the photo-degradation of TPB coatings has not been quantified as a function of a VUV exposure comparable to that obtained in a noble gas detector, operated in the conditions of an underground experiment.

In this paper, we present a study of TPB ageing due to exposure to VUV light around $170 \mathrm{~nm}$ (xenon scintillation peak), aiming at quantifying the reduction of the absolute fluorescence yield (or conversion efficiency) of TPB coatings as a function of exposure in the gaseous xenon TPC used by the NEXT experiment for $\beta \beta$ decay search. Several TPB coatings of different thicknesses ( $130 \mathrm{~nm}, 260 \mathrm{~nm}, 390 \mathrm{~nm}, 1600 \mathrm{~nm}$ ) were deposited by vacuum evaporation on quartz substrates, following the protocol described in [6]. Each sample was exposed to controlled VUV light in a dedicated setup, which simulates the exposure conditions in the gaseous Xe electroluminescent TPC, called NEW, used in the NEXT experiment [3]. This TPC is presently being installed in the Canfranc Underground Laboratory (LSC) [7] for measuring the two-neutrino $\beta \beta$ decay mode $(\beta \beta 2 \nu)$ of the 136Xe isotope. The absolute quantum yield of the TPB-coatings was measured before and after exposure. The photo-degradation of the TPB resulting from this VUV exposure is analyzed and discussed.

\section{Experimental setup and method}

Several rectangular quartz samples of dimensions $15 \times 10 \times 1 \mathrm{~mm}^{3}$ were used for the vacuum deposition of TPB layers of different 


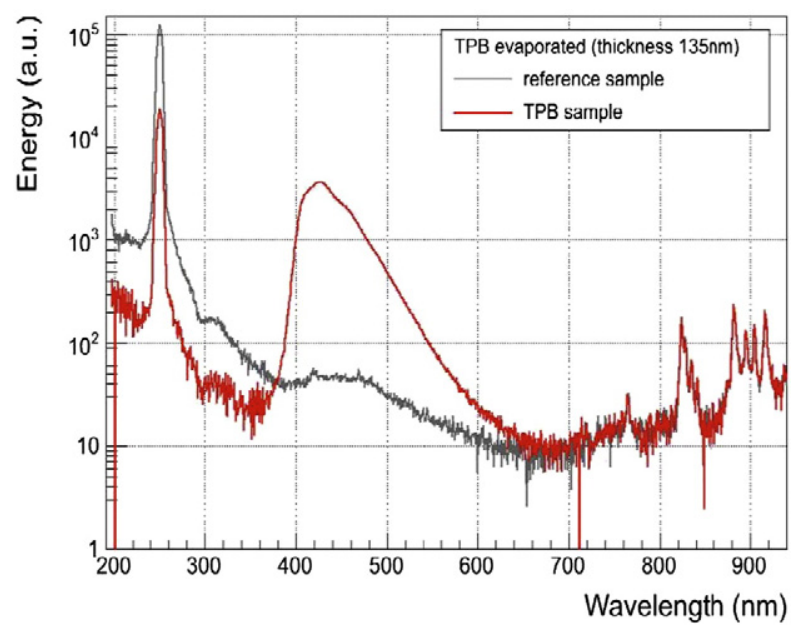

Fig. 1. Fluorescence spectrum (red curve) obtained exciting a TPB layer, deposited on a quartz substrate, with UV light at $250 \mathrm{~nm}$ (black curve) from a Xe lamp. The excitation spectrum (black) is obtained using a reference quartz substrate without TPB.

thicknesses: $130 \mathrm{~nm}, 260 \mathrm{~nm}, 390 \mathrm{~nm}$ and $1600 \mathrm{~nm}$. The absolute quantum yield (or fluorescence efficiency) of these coatings was measured immediately after the coating process and after exposure to VUV light following the procedure described in the next subsections. These samples, when not exposed to VUV light, were stored in dark and in nitrogen atmosphere to avoid hydration and oxidation that may bias our photo-degradation measurements.

\subsection{VUV exposure setup and method}

The photo-degradation of TPB coatings and the subsequent reduction of the fluorescence resulting from exposure to light is a stochastic process that depends on the light intensity $I$, its frequency or event rate $\nu$ and exposure time $T$. The light intensity may be expressed in terms of photon flux, $\phi=I \nu / \epsilon$, where $\epsilon$ is the photon energy. The exposure $E$ can then be defined by Eq. (1).

$E=I \nu T=\phi \epsilon T$

In a noble gas TPC, the interaction of a particle with the gas produces scintillation and ionization. The ions and free electrons produced have to be drifted, amplified and read out for the detection of the particle. The electroluminescence (EL) is an optical amplification process of the ionization signal [8]. It is produced by drifting and accelerating the electrons in a narrow region of high electric field, where secondary scintillation light is generated, as shown in Fig. 2, with a gain of about 2 thousand photons per electron (see page 3 reference [9]). The EL yield thus depends on the energy of the incident particles that originate the ionization charges and on the reduced electric field used to accelerate the electrons, typically 2 to $3 \mathrm{kV} / \mathrm{cm} /$ bar. In argon and xenon, the EL scintillation is emitted in the VUV spectral region, around $128 \mathrm{~nm}$ and $172 \mathrm{~nm}$, respectively.

In order to assess the degradation of the TPB-coatings in such a TPC over long periods of time (at least several months), as usually required for underground experiments, an equivalent light exposure has to be provided on similar TPB-coatings, using a monochromatic VUV light of fixed intensity and frequency, varying the exposure time, to be compared to the exposure obtained in the TPC over time.

In our test setup (Fig. 3), VUV light is provided by a deuterium lamp coupled to a vacuum monochromator (VMC) for the selection of the irradiation wavelength at $170 \pm 3 \mathrm{~nm}$. The VMC output is coupled to the vacuum chamber containing the TPB-coated quartz samples to expose to this monochromatic VUV light. A vacuum level close to $10^{-4} \mathrm{mbar}$ is obtained using a primary pump in series with two turbo-molecular pumps, respectively connected to the vacuum chamber and to the VMC. A fused silica diffusor lens with 220 grit polishes (Thorlabs DGUV10-220) is used at the VMC output to provide a uniform illumination of the TPB-coated sample. This latter is placed a few $\mathrm{cm}$ away from the light source, at a fixed distance, where the light intensity is previously measured by a calibrated photomultiplier tube (PMT) operated without gain, i.e. with the current taken at the first dynode. The PMT photocurrent provides the direct measurement of the photon flux at the chosen longitudinal distance from the VMC output, where the TPB samples are subsequently placed. The PMT and sample supports are fixed to a movable vacuum feed-through that allows the correct adjustment of the PMT/sample position to the desired light intensity. The PMT is removed from the chamber for the successive VUV irradiation of the TPB samples.

The energy of the VUV radiation (at $170 \pm 3 \mathrm{~nm}$ ) being similar in both the Xe TPC (Fig. 2) and the test setup (Fig. 3), from Eq. (1) we can set the condition of normalization of the exposure in the TPC and in the test setup:

$\phi_{1} T_{1}=\phi_{2} T_{2}$

In Eq. (2), $\phi_{1}$ and $\phi_{2}$ are the photon fluxes (number of photons per unit of time and per unit of area) impinging in the TPB-coated surfaces for the TPC and the test setup, respectively; $T_{1}$ and $T_{2}$ are the respective exposure times.

In order to determine the VUV light exposure in the TPB-coated surfaces of the NEXT TPC presently being installed at LSC (NEW), we assume a maximum event rate of $100 \mathrm{~Hz}$ in the two-neutrino $\beta \beta$ decay spectral region and an average energy of $1 \mathrm{MeV}$ for the $\beta \beta$ events. We assume an electric field of $2 \mathrm{kV} / \mathrm{cm} / \mathrm{bar}$ in the TPC EL region, which corresponds to an optical gain of about 2000 photons per primary electron [9]. The average energy required to produce an electron-ion pair in xenon being $W=24.8 \mathrm{eV}$, the number of primary electrons produced per event of $1 \mathrm{MeV}$ is $10^{6} / 24.8=40323$. As a result, the total number of EL photons per event of $1 \mathrm{MeV}$ is $N_{1}=8.064 \times 10^{7}$.

In order to determine the flux of photons in the TPB-coated surfaces of the NEW TPC, we need the dimensions of the chamber active volume. NEW has a cylindrical field cage with a length $L=508 \mathrm{~mm}$ and a radius $R=221 \mathrm{~mm}$. Since all the TPC inner surfaces are covered with TPB, the total area covered is $A_{1}=2 \pi R^{2}+2 \pi R L=1.012 \times 10^{6} \mathrm{~mm}^{2}$. The flux of photons in the TPB layers is then $\phi_{1}=N_{1} \nu_{1} / A_{1}=7967$ photons $/ \mathrm{s} /$ $\mathrm{mm}^{2}$, assuming an event rate $\nu_{1}=100 \mathrm{~Hz}$.

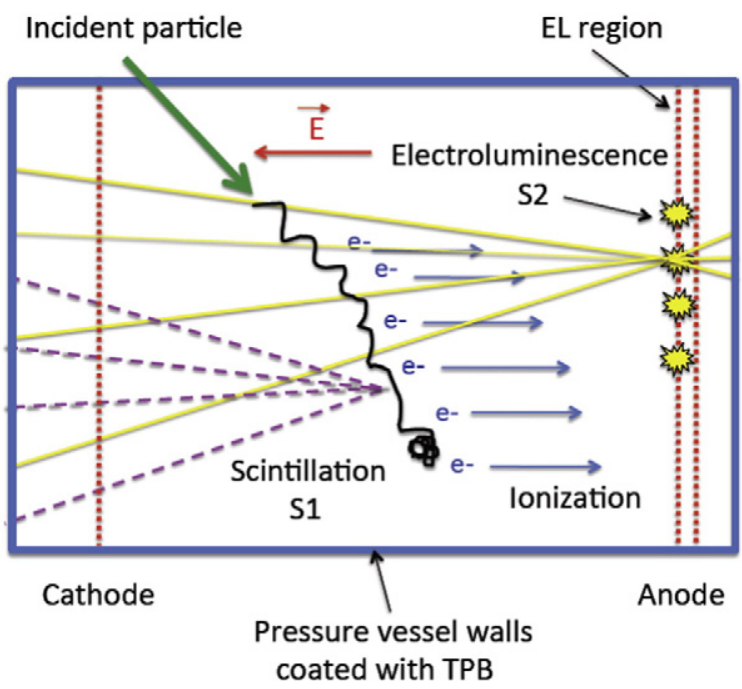

Fig. 2. Electroluminescence process in a noble gas TPC. The EL signal (S2) amplifies the ionization signal (electrons) drifted to the anode. 


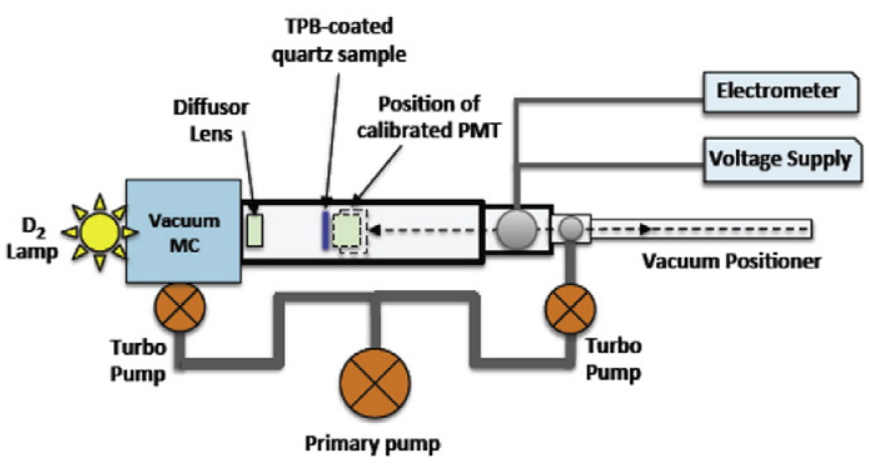

Fig. 3. Experimental setup used to assess the degradation of TPB-coating samples exposed to VUV light at $170 \pm 3 \mathrm{~nm}$ from a deuterium lamp coupled to a vacuummonochromator for the selection of the wavelength. The PMT is used for the calibration of the VUV light intensity prior to the irradiation of the TPB samples.

To determine the flux of VUV light at $170 \mathrm{~nm}$ in the test setup (Fig. 3), we use the photocurrent measured in the PMT at the position of the TPB samples, which is $1 \mathrm{nA}$, i.e. $6.242 \times 10^{9}$ electrons collected per second. Taking into account the quantum efficiency of the calibrated PMT ( 0.3132 at $170 \mathrm{~nm}$ provided by the manufacturer), the number of VUV photons impinging in the PMT is $19.93 \times 10^{9}$ per second. The PMT active area is $A_{2}=420.5 \mathrm{~mm}^{2}$. As a result, the VUV photon flux in the PMT is $\phi_{2}=N_{2} \nu_{2} / A_{2}=19.93 \times 10^{9} / 420.5=4.74 \times 10^{7}$ photons $/ \mathrm{s} /$ $\mathrm{mm}^{2}$. The flux in the TPB-coated samples is the same as they are placed at the position where the PMT surface was placed for calibration.

For an exposure time of 1 month in the TPC $\left(T_{1}=2.592 \times 10^{6} \mathrm{~s}\right)$, according to Eq. (2) the equivalent exposure time in the test setup is $T_{2}=$ $T_{1} \phi_{1} / \phi_{2}=436 \mathrm{~s}$ (or $7.3 \mathrm{~min}$ ). So, irradiating the TPB-coated samples over $7 \mathrm{~min}$ in our test setup, with a photon flux corresponding to $1 \mathrm{nA}$ current in the PMT, would correspond to 1 month of exposure to VUV light in the NEW TPC.

\subsection{Absolute quantum yield measurement}

The absolute quantum yield (QY) of a TPB-coating is the ratio between the intensity of the fluorescence light emitted by the coating and the excitation light absorbed. To obtain the fluorescence spectrogram of a TPB-coated quartz sample, the sample was placed inside an integration sphere coupled to a spectrometer, within which it was exposed to monochromatic UV-visible light. The quantum yield was then measured from the spectrogram.

A scheme of the experimental setup used is shown in Fig. 4. It includes a xenon lamp from Hamamatsu Photonics (model E7536, $150 \mathrm{~W}$ ), coupled to a monochromator (MC) for the selection of the excitation wavelength, and a spectrometer also from Hamamatsu Photonics (Multichannel Analyzer C10027) coupled to an integration sphere from Spectralon. The sphere is internally covered with a diffuse and highly reflective PTFE coating, allowing multiple scattering of the light, whose intensity is equal at any point of the sphere. The light is conducted from the MC to the integrating sphere and from this latter to the spectrometer through quartz optical fibers of $1 \mathrm{~mm}$ diameter.

The spectrogram of the coated sample is measured and compared to that of an uncoated reference quartz sample of the same dimensions which provides the excitation spectrogram as shown in Fig. 1. The absolute quantum yield of the TPB layer is calculated as the ratio between the fluorescence yield and the absorption yield, the latter being determined as the difference between the incident and scattered light peaks as shown in Fig. $5, \mathrm{~S}_{0}$ being the area of the incident light peak, $S_{1}$ the area of the light peak scattered from the sample, and $S_{2}$ the area of the light peak emitted by the sample. QY is determined from Eq. (3). The excitation wavelengths considered were from $250 \mathrm{~nm}$, which is the lowest wavelength measured by the spectrometer, up to $430 \mathrm{~nm}$ which corresponds to the fluorescence peak of the TPB.

$Q Y=\frac{S_{2}}{S_{0}-S_{1}}$

The duration of the exposure to UV-visible light of the TPB-coating samples for the QY measurement is negligible (typically 3 to $4 \mathrm{~s}$ ) when compared to the minimum exposure time (between $7 \mathrm{~min}$ and $\sim 23 \mathrm{~h}$ ) used for the photo-degradation studies. Also, the exposure to the air and ambient light of the samples did not exceed a few minutes necessary for the QY measurement. The operation conditions and measurement time of the QY have thus a negligible influence on the

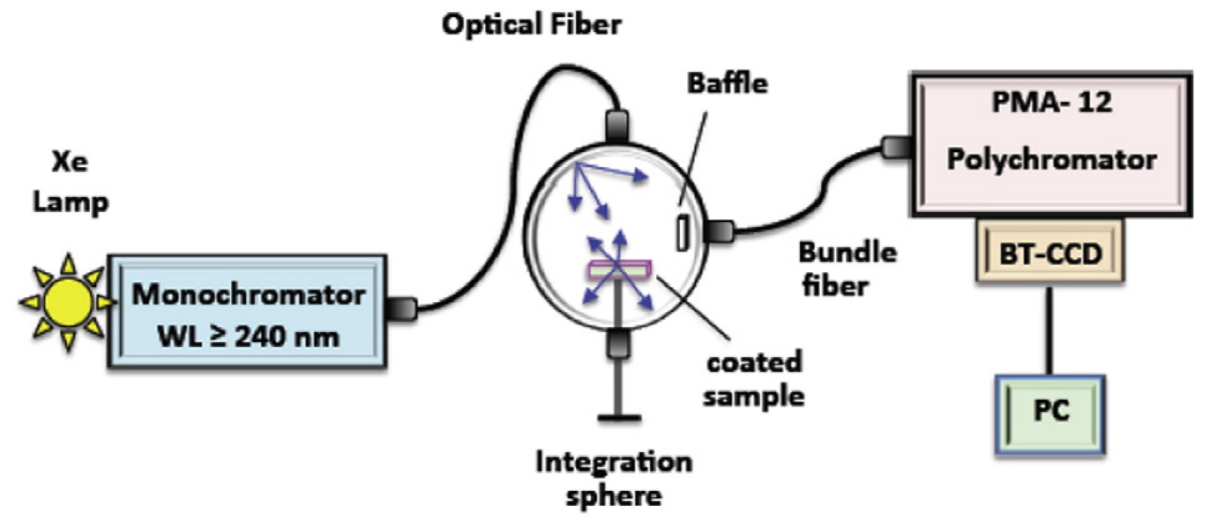

Integration sphere:

Internal surface spectralon : $98 \%$

Reflectance at $250 \mathrm{~nm}$

PMA-12 : Photonic Multichannel Analyzer (Hamamatsu)

Polychromator : Czerny Turner type

BT-CCD : back-thinned CCD image sensor (Hamamatsu)

PC : Personal Computer

Fig. 4. Experimental setup used to measure the quantum yield of different TPB-coating samples. 


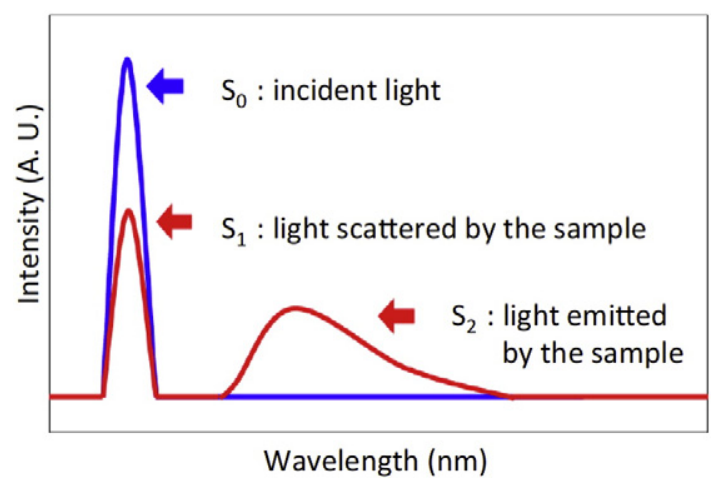

Fig. 5. QY measurement principle based on the absorption and emission spectrograms from the samples.

irradiation effects on the TPB produced at $170 \mathrm{~nm}$ in the VUV exposure setup (Fig. 3).

However, the variation in the exposure time of the samples (between 1 and $12 \mathrm{~s}$ ) that may be set manually in the integration sphere for QY measurements, induce fluctuations in the QY value at the level of $1.2 \%$. For a given time setting, QY is calculated as the mean value of 20 measurements provided automatically by the spectrometer software. In order to assess the additional uncertainties introduced by the measurement conditions and the operator, we have evaluated the statistical uncertainty on this mean QY value from a set of 10 repeated measurements, at a fixed wavelength and time exposure settings. The relative standard deviations obtained are of $1.06 \%$ and $0.33 \%$ for the $130 \mathrm{~nm}$ thick and $1600 \mathrm{~nm}$ thick TPB samples respectively.

The effects of the ambient temperature and the calibration of the spectrometer were also considered for the assessment of the systematic uncertainties on the QY values. Ambient temperature has no effect on the QY as this magnitude is determined as a ratio of light yields, which cancels possible variations due to temperature. The calibration of the spectrometer was checked using various reference fluors provided by Hamamatsu. Finally, the combined relative uncertainties on the QY values presented in this paper are $1.6 \%$ and $1.2 \%$ for the $130 \mathrm{~nm}$ thick and $1600 \mathrm{~nm}$ thick TPB coatings respectively.

\section{Results}

For each TPB-coating sample, we measured first the quantum yield as a function of wavelength, in the range $250 \mathrm{~nm}$ up to $430 \mathrm{~nm}$, using the setup shown in Fig. 4 and according to the method described in Fig. 5. The results are shown in Fig. 6.

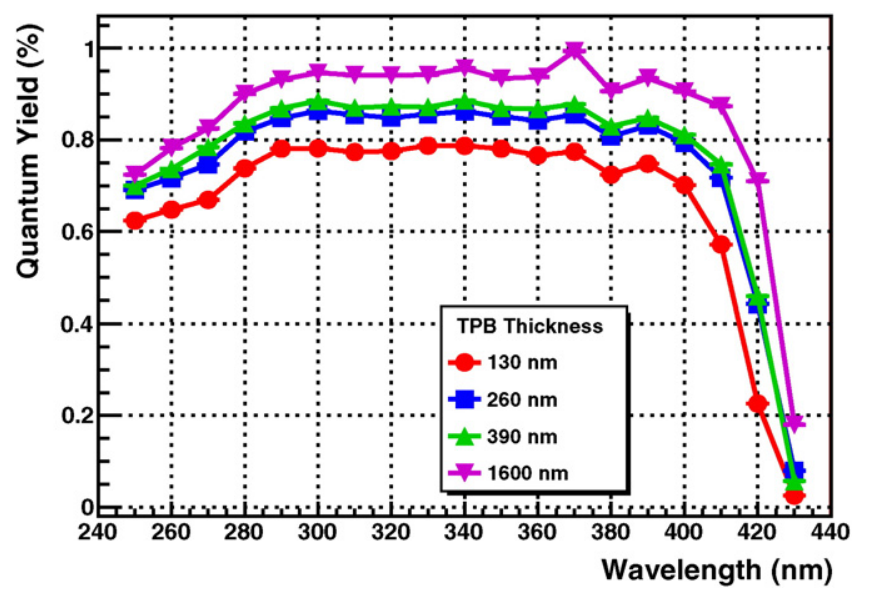

Fig. 6. Quantum yield of several TPB coating samples with different thicknesses as a function of excitation wavelength in the region $250-430 \mathrm{~nm}$, before exposure to VUV light.
Then we moved the samples to our VUV light exposure setup, shown in Fig. 3, to be irradiated by different periods of time. After each period of irradiation, the quantum yield of the samples was measured as a function of wavelength using the setup of Fig. 4. This study was made for the thinner $(130 \mathrm{~nm})$ and the thicker (1600 nm) TPB-coating samples.

\subsection{Quantum yield variation with coating thickness}

Fig. 6 shows the quantum yield measured as a function of the excitation wavelength for several TPB-coating samples with different thicknesses $(130,260,390,1600 \mathrm{~nm})$, before irradiation with VUV light $(170 \mathrm{~nm})$. As seen in the figure, the quantum yield or fluorescence efficiency increases with TPB coating thickness, being close to $95 \%$ in the range $300-370 \mathrm{~nm}$ for the thickest coating of $1600 \mathrm{~nm}$. No significant dependence of the quantum yield on the excitation wavelength is observed in the UV range $300-370 \mathrm{~nm}$, while above $400 \mathrm{~nm}$ there is a clear drop of the efficiency vanishing around $430 \mathrm{~nm}$. TPB is indeed transparent to its fluorescence light (peak at $430 \mathrm{~nm}$ ), as no absorption is observed at the excitation wavelength of $430 \mathrm{~nm}$. To compare our QY values to available fluorescence efficiency measurements reported in the literature, we consider the fluorescence efficiency measured in reference [10] for a TPB coating similar to our thickest sample. An efficiency close to $80 \%$ is reported at $250 \mathrm{~nm}$, to be compared to our QY of $72 \%$. These results are compatible at the considered wavelength, considering the uncertainties and the different measurement methods used.

\subsection{Quantum yield after exposure to VUV light}

The TPB-coated samples with thicknesses of $130 \mathrm{~nm}$ and $1600 \mathrm{~nm}$ were exposed to VUV light, using the setup of Fig. 3, for different periods of time. After each irradiation the quantum yield of the samples was determined as a function of excitation wavelength. Figs. 7 and 8 present the results obtained for the thinner sample $(130 \mathrm{~nm})$ and the thicker sample $(1600 \mathrm{~nm})$ respectively. The equivalence between exposure times in the test setup and in the NEW TPC is shown for every curve in these figures.

As shown in Figs. 7 and 8, the variations in the quantum yield values as a function of excitation wavelength after long exposure time to VUV light are large. At short excitation wavelengths (250-290 nm), the degradation of $\mathrm{QY}$ produced at the first VUV exposure (equivalent to 1 month in the TPC) does not increase significantly with much larger exposure times and seems to saturate. This behavior is observed for both samples, the thin and the thick one, being the degradation, at the first

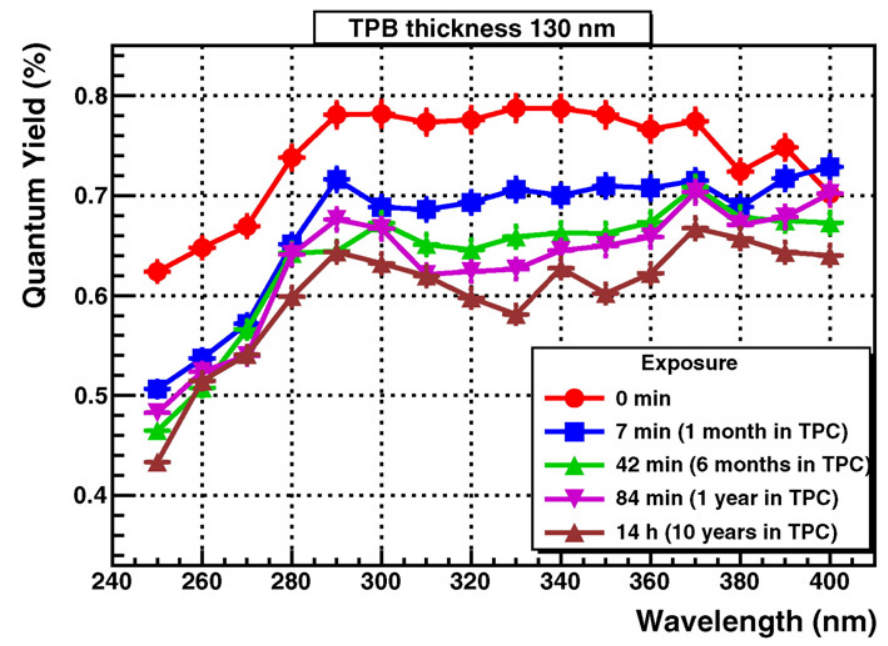

Fig. 7. Quantum yield as a function of wavelength for the TPB coating sample of $130 \mathrm{~nm}$ thickness, after different exposure times to VUV light. Equivalence to the exposure in the NEW TPC is shown. 


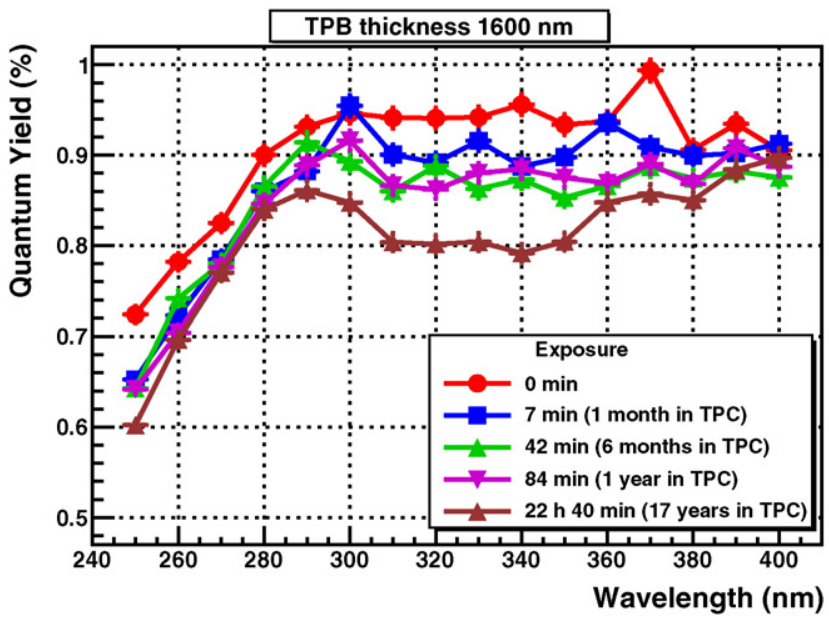

Fig. 8. Quantum yield as a function of wavelength for the TPB coated sample with $1600 \mathrm{~nm}$ thickness, after different exposure times to VUV light. Equivalence to the exposure in the NEW TPC is shown.

exposure, larger for the thin sample $(\sim 18 \%)$ than for the thick one $(\sim 10 \%)$. At longer excitation wavelengths, in the region $300-350 \mathrm{~nm}$, QY shows more significant dependence with exposure time, being the reduction larger for the thin sample than for the thick one at the same exposure time. For the thin sample, the reduction of QY is up to 20\% at 1 year exposure time in the TPC and $28 \%$ at 10 years exposure time in the TPC, while the reduction level of QY in the thick sample reaches $\sim 18 \%$ only after 17 years exposure time in the TPC.

The measurements shown in the plots of Figs. 7 and 8 have been performed in July 2015. Since then, the TPB samples were stored in dark and $\mathrm{N}_{2}$ atmosphere.

The QY measurements of the two TPB samples irradiated at $170 \mathrm{~nm}$

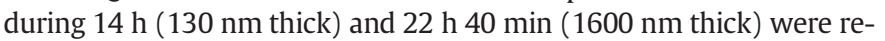
peated 7 months later (February 2016). The relative mean variation of the QY values obtained after this long time storage was $4 \%$ for the thin coating and $2 \%$ for the thick one. This result indicates that within the measurement uncertainties described in Section 2.2 and the modifications of the samples related to the 7 months storage time, the results shown in the QY plots presented in this paper are quite reliable. In addition, this shows that TPB samples, stored in appropriate conditions (absence of light, oxygen and humidity), may well preserve their fluorescence efficiency over time.

\section{Conclusions and discussion}

In this work, we present studies of the fluorescence degradation of TPB coatings due to prolonged and controlled exposure to VUV monochromatic light at $170 \pm 3 \mathrm{~nm}$ (similar to the scintillation of Xe). This work aims at quantifying the reduction of the absolute quantum yield (or fluorescence efficiency) of thin (130 nm) and thick (1600 nm) TPB coatings exposed to VUV light doses comparable to those expected in a real electroluminescent Xe TPC, operated during periods of time ranging from 1 month to several years.

The absolute quantum yield of TPB coating samples with different thicknesses ( $130 \mathrm{~nm}, 260 \mathrm{~nm}, 390 \mathrm{~nm}, 1600 \mathrm{~nm}$ ), was found to increase with the coating thickness, being close to 95\% in the UV spectral range $300-370 \mathrm{~nm}$ for the thickest coating (1600 nm), while above $400 \mathrm{~nm}$ a clear drop of the efficiency is observed, vanishing around $430 \mathrm{~nm}$. TPB is indeed transparent to its fluorescence light, as no absorption is observed at the excitation wavelength of $430 \mathrm{~nm}$.

The variation of the quantum yield for prolonged exposure time of the thinner and thicker samples to VUV light at $170 \mathrm{~nm}$ was investigated. The exposure in our test setup was normalized to the one expected in the NEW TPC, used in the NEXT experiment, so that an exposure of
$84 \mathrm{~min}$ in our test setup, for instance, is equivalent to 1 year exposure in the TPC in normal continuous operation conditions.

The quantum yield as a function of the excitation wavelength, measured right after the VUV irradiation of the TPB samples, shows an irregular behavior which contrasts with the smooth regular behavior before irradiation. The quantum yield as a function of the exposure time to VUV light appears to depend significantly on the wavelength used for the excitation of the molecule and the characterization of its fluorescence mechanism. The behavior observed is not an artifact of our QY measurement procedure and related uncertainties, as these have been accurately analyzed and evaluated, and found to correspond to $<2 \%$. The large variation of the QY with wavelength observed, seems to suggest it is related to the molecular states immediately after irradiation. It may also indicate that the VUV light damages only part of the molecular structure responsible of the wavelength shifting mechanism. This damage would induce an irregular absorption/reemission pattern with wavelength.

The variation of QY with exposure times of the thin and thick TPB samples, highlighted by the excitation light in the range $300-350 \mathrm{~nm}$, is not significantly observed at short excitation wavelengths in the range 250-290 nm. This feature, most probably related to the molecular structure of the irradiated coatings, would suggest a possible similar behavior of QY at VUV excitation wavelengths. The fluorescence efficiency of $\sim 80 \%$ at $250 \mathrm{~nm}$ and $170 \mathrm{~nm}$ reported in reference [10], for a coating similar to our thick one, may suggest that the fluorescence mechanism at these two excitation wavelengths are similar, and therefore, we would expect a similar fluorescence response after prolonged irradiation, i.e., $<15 \%$ VUV-induced reduction of QY after about 5 years continuous operation of the TPC.

It was not possible with the QY measurement setup described in this paper, to assess the QY degradation of the irradiated TPB samples when excited in the VUV spectral range, and especially at the Xe scintillation wavelength $(170 \mathrm{~nm})$. A quantum yield measurement equipment suitable for vacuum ultraviolet light should be used in this case. Such equipment is not available for us at present.

However, with the measurements presented in this paper, in which the TPB samples are irradiated at VUV doses comparable to those expected in a real noble gas detector, and then illuminated in the UVvisible for assessing the variation in the fluorescence mechanism, we provide a magnitude of the damages in the fluorescence yield that may be expected in the TPC over large exposure times. This damage, due only to exposure to monochromatic VUV light, is shown not to exceed $15 \%$ in the lifetime and real operation conditions of the underground experiment, typically 5 years operation with an event rate lower than $100 \mathrm{~Hz}$. The thick TPB coating $(1600 \mathrm{~nm})$ has shown to have larger fluorescence efficiency, between $95 \%$ and $100 \%$, and stronger resistance to VUV radiation damage than the thin coating $(130 \mathrm{~nm})$.

Finally, it is here worth to compare our study with previous ones reported in the literature and addressing the TPB photo-degradation, mainly references [5] and [11], in which TPB samples are exposed to direct sunlight, and to a full spectrum fluorescent light shone through various filters, in air, and during several hours. Severe degradation of the TPB samples has been reported, with potential losses at up to $80 \%$ level after a month of exposure. In these reported works, the light doses to which the samples are exposed are much larger than the largest dose used in our studies, which compares to that expected in a real electroluminescent TPC in operation during several years. The conclusions on the strong degradation reported do not address the fluorescence degradation as a function of the exposure dose to monochromatic VUV light, i.e., in similar conditions as in a noble gas TPC.

In the present paper, despite the limitations of our setup for a complete characterization of the irradiated TPB samples in the VUV spectral range, a method for a quantification of the quantum yield reduction induced by exposure to VUV light is presented, and an assessment of the order of magnitude of the reduction of the TPB fluorescence yield in a noble gas TPC, after several years of operation, is provided. 
By repeating our QY measurements of the TPB samples irradiated at $170 \mathrm{~nm}$ after 7 months storage of the samples in a controlled atmosphere (dark and $\mathrm{N}_{2}$ ), we found that no $>4 \%$ average relative variation is obtained on QY. This indicates both the reliability of our measurements and the stability of the coatings stored in appropriate conditions.

As indicated in reference [11], TPB coatings prepared for their use in a noble gas TPC, for Dark Matter or neutrinoless double beta decay experiments, have to be preserved from humidity, oxygen, sunlight and strong UV exposure. However, in-vessel VUV-induced degradation of coatings of $\sim 1.6 \mu \mathrm{m}$ thickness, does not seem to be a major issue in the normal irradiation and time exposure conditions of the underground experiments.

\section{Acknowledgements}

We acknowledge the NEXT Collaboration and the European Research Council (ERC) for the Advance Grant 339787-NEXT; the Spanish Ministry of Economy and Competitiveness (MINECO) via the Unidad de Excelencia María de Maeztu MDM-2015-0538 and MAT2014-55200, and the Severo Ochoa program under contract SEV-2014-0398, and for the research grants under contracts CSD2008-00037 (Consolider Ingenio 2010), FIS2012-37947-C04-03, part of which comes from FEDER funds; and the Generalitat Valenciana (Prometeo/2012/053).

N. Yahlali acknowledges the Spanish MINECO for the research grant under contract FPA2014-61149-JIN, part of which comes from FEDER funds.
L.M.P. Fernandes acknowledges support from FEDER, through program COMPETE, and National funds through FCT in the frame of project PTDC/FIS/0843/2012, F-COMP-01-0124-FEDER-029179.

We also thank Manolo at the IFIC workshop for his dedication and $\mathrm{H}$. Bolink, A. Lopez and A. Pertegas from ICMOL for their valuable collaboration.

\section{References}

[1] Elena Aprile, Aleksey E. Bolotnikov, Alexander I. Bolozdynya, and Tadayoshi Doke, Noble Gas Detectors. WILEY-VCH Verlag GmbH \& Co. KGaA.

[2] http://www.sigmaaldrich.com.

[3] J.J. Gómez-Cadenas, et al., Present status and future perspectives of the NEXT experiment, Adv. High Energy Phys. 2014 (2014) 907067.

[4] R. Francini, et al., Tetraphenyl-butadiene films: VUV-vis optical characterization from room liquid argon temperature, J. Instrum. 8 (2013), C09010.

[5] B.J.P. Jones, et al., Photodegradation mechanisms of tetraphenyl butadiene coatings for liquid argon detectors, J. Instrum. 8 (2013), P01013.

[6] V. Álvarez, et al., SiPMs coated with TPB: coating protocol and characterization for NEXT, J. Instrum. 7 (2012), P02010.

[7] http://www.lsc-canfranc.es/en/

[8] L.M.P. Fernandes, et al., Primary and secondary scintillation measurements in a xenon gas proportional scintillation counter, J. Instrum. 5 (2010), P09006.

[9] C.M.B. Monteiro, et al., Secondary scintillation yield in pure xenon, J. Instrum. 2 (2007), P05001.

[10] V.M. Gehman, et al., Fluorescence efficiency and visible re-emission spectrum of tetraphenyl butadiene films at extreme ultraviolet wavelengths, Nucl. Instrum. Methods Phys. Res. A654 (2011) 116.

[11] C.S. Chiu, et al., Environmental effects on TPB wavelength-shifting coatings, J. Instrum. 7 (2012), P07007. 